\title{
The proton radius: are we still puzzled?
}

\section{Evangeline J. Downie ${ }^{* \dagger}$}

The George Washington University

E-mail: edownie@gwu.edul

The proton radius puzzle began in 2010 when the CREMA Collaboration released their measurement of the proton radius [1] from muonic hydrogen spectroscopy: $r_{p}=0.84184(67) \mathrm{fm}$, This was five standard deviations smaller that the accepted CODATA value of that time $(0.8768(69) \mathrm{fm})$ [2], and sparked an enduring and intriguing puzzle.

Since that first measurement, many theories have been formed as to why the proton radius appears smaller when measured with muons than it does when measured with electrons. Many new measurements have also been made in an attempt to resolve the puzzle. We will detail the current status of the proton radius puzzle, and review future experimental plans, with a focus on the MUon proton Scattering Experiment (MUSE).

The 9th International workshop on Chiral Dynamics

17-21 September 2018

Durham, NC, USA

* Speaker.

${ }^{\dagger}$ On behalf of the MUSE Collaboration 


\section{Introduction}

The proton radius puzzle (PRP) began in 2010 when the CREMA collaboration published their measurement of the charge radius of the proton, which is defined as:

$$
r_{p}^{2}=-\left.6 \frac{d G_{e}\left(q^{2}\right)}{d q^{2}}\right|_{q=0}
$$

where $r_{p}$ is the mean charge radius of the proton, $G_{e}(q)$ is the Sachs electric form factor, and $q$ is the momentum transfer. The radius, extracted from spectroscopy of muonic hydrogen, was reported as $r_{p}=0.84184(67) \mathrm{fm}$ [1], more than five standard deviations from the accepted CODATA value of $r_{p}=0.8768(69) \mathrm{fm}$ [2], but an order of magnitude more precise. This resulted in a flurry of calculations, publications, and experimental proposals which, thus far, have not resolved the puzzle. Indeed, in many cases, they have made the puzzle somewhat more puzzling. We review the different mechanisms of measuring the proton charge radius, examine the status of the PRP, potential solutions, and the experiments planned to resolve it.

\section{Electron scattering measurements}

The first measurement to extract the radius was reported in 1956 [3]. Chambers and Hofstadter used electron scattering off a gaseous hydrogen target to determine a radius of $r_{p}=0.77(10) \mathrm{fm}$. Since then, accelerators and detectors have improved, and further developments have been made in theory and radius extraction techniques leading to increasingly precise determinations of the proton radius. In 2010, Bernauer et al. [4] reported the largest data set in elastic-proton scattering measured to date at MAMI. Shortly thereafter, Zhan et al. [5] reported on an extraction of $G_{E} / G_{m}$ over a wide $q^{2}$ from JLab. Both Bernauer and Zhan supported the larger electric charge radius reported in the CODATA 2010 average of $r_{p}=0.8768(69) \mathrm{fm} \mathrm{[2].}$

There are several weaknesses in the extraction of the charge radius from scattering experiments. The scattering measurements produce cross sections, which are then used to extract form factors. The resulting form factors are plotted as a function of $q^{2}$, and fitted to extract a radius. This primary issue is that it is not possible to measure exactly at $q^{2}=0$, as there no scattering actually occurs, thus radius extractions are always interpolating outside of the measured range. If one measures at very low $q^{2}$, one must measure incredibly precisely to see the effect of the radius, as the effect of the radius becomes very small at lower $q^{2}$ (see Figure 1). If one measures only over a very limited, low $q^{2}$, range, one might miss higher order effects, resulting in truncation error on the extracted radius, which can be as large as the 4\% PRP effect [6].

Thus scattering measurements must cover a range of $q^{2}$ values, reaching as low as possible, but extending far enough out from zero in order to minimize truncation error [6]. There are also issues in the extraction of the radius by fitting: depending on the parameterization used, one can extract both large and small radii from the same data set, as can be seen from the many extractions reported at the 2018 MITP workshop in Mainz [8]. 


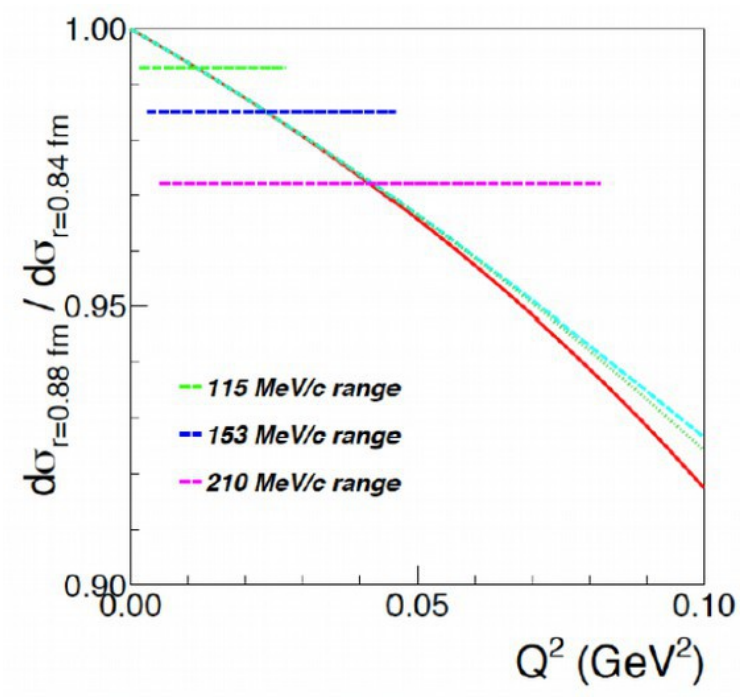

Figure 1: Effect on the cross section from the proton radius being $0.88 \mathrm{fm}$ vs. $0.84 \mathrm{fm}$. The solid red line uses a linear approximation, $G_{E}=1-Q^{2} r^{2} / 6$. The cyan dashed and green dotted curves include $Q^{4}$ and $Q^{6}$ terms taken from the Kelly parameterization, respectively. The horizontal green, blue, and magenta dashed lines indicate the kinematic range of the proposed MUSE data. Figure from [7].

\section{Spectroscopy Measurements}

\subsection{Hydrogen Spectroscopy}

There is another mechanism by which one can extract the proton radius: hydrogen spectroscopy. There is a small perturbation to the S-orbitals, caused by the finite radius of the proton. The finite size of the proton has, however, no impact upon the P-orbitals, as the wavefunction of electrons in $\mathrm{P}$ orbitals is zero at the origin. This means that there is a small perturbation to the S-P orbital transition in hydrogen, around $0.014 \%$ the size of the Lamb Shift. Aside from this radius perturbation, all other components of the difference are precisely calculable in QED. Thus, due to the high precision with which lasers can be tuned and calibrated, it is possible to extract the radius of the proton from a measurement of S-P transitions within atomic hydrogen. This measurement, too, has been performed at multiple institutions, with ever-increasing precision, and, until the inception of the PRP, it also agreed with the CODATA 2010 average [2]. For a full review of measurements prior to the PRP, and the immediate impact of the PRP, see $[9,10]$.

\subsection{Muonic Hydrogen Spectroscopy}

While there were two different measurement mechanisms, with completely different systematics, which produced the same proton radius, the uncertainty on the proton charge radius was still the dominant uncertainty in many QED processes. In an effort to improve this situation, the CREMA collaboration decided to measure the radius using muonic hydrogen, hydrogen where the electron has been replaced by the muon. As the muon is $\sim 207$ times as massive as the electron, muonic hydrogen is far more compact than atomic hydrogen. This makes the perturbation to the 
S-orbitals far greater than that in atomic hydrogen, and therefore the radius extraction from muonic hydrogen is roughly eight million times more sensitive to $r_{p}$.

The CREMA collaboration successfully extracted the proton charge radius from their data, reporting in 2010 a radius of $r_{p}=0.84184(67) \mathrm{fm}$ [1]. This is an order of magnitude more precise than all proior measurements, but $4 \%$ smaller, and, at that time, in roughly $5 \sigma$ disagreement with the accepted value of CODATA.

The CREMA collaboration continued to work on the proton radius, publishing again in 2013 a report with further analysis of the data, including an extraction of the magnetic radius. Antognini et al. published a value of $r_{p}=0.84087(39) \mathrm{fm}$ [11]. This was then $7.9 \sigma$ from the 2010 CODATA value.

\subsection{Muonic Deuterium and Helium Spectroscopy}

In order to further investigate the radius problem, CREMA moved to investigating the radius of deuterium, helium and other nuclei. In 2016, they published the results of the deuterium measurements, with an extracted radius of $r_{d}=2.12562(78) \mathrm{fm}$ [12], which was almost three times more precise than the CODATA 2010 value of $r_{d}=2.1424(21) \mathrm{fm}$, but $7.5 \sigma$ smaller. Using the well-known isotope shift, the muonic deuterium radius also gave a proton radius in agreement with the smaller muonic hydrogen radius.

At the MITP workshop in the summer of 2018 [8], it was reported, however, that the measurements performed on various muonic helium agreed with the radii from atomic helium. Thus we were forced to acknowledge that this is an $Z=1$ radius problem.

\section{Recent developments}

In addition to the progress in muonic hydrogen, there have been multiple efforts to make more precise measurements of the proton charge radius using atomic hydrogen spectroscopy. This has resulted in mixed outcomes, with Beyer et al. extracting a radius in agreement with the smaller muonic hydrogen proton charge radius [13], while Fleurbaey extracted a larger radius [14].

Additionally, several experiments are underway, or under analysis, to measure the radius via electron scattering at exceptionally low $q^{2}$. PRad at Jefferson Lab [15] gave a preliminary report at this meeting, and so we will not go into more detail here. At PRAE in Orsay, there are also plans to have a low- $q^{2}$ experiment, known as ProRad [16]. An effort at MAMI to reach even lower $q^{2}$ using Initial State Radiation showed that the methodology worked in principle, but needs much higher statistics to be competitive, thus it is planned to repeat this experiment at the MESA accelerator, which is currently under construction [17].

To date, results remain divided between the smaller and larger radius, and, in some cases, multiple extractions from the same data set give different answers. Even were all of the newer results to settle on one radius, it would still be necessary to explain the cause of the discrepancy in the existing data [8].

\section{Potential resolutions to the Proton Radius Puzzle}

Since the inception of the puzzle in 2010, many different theories have been postulated to 
explain the puzzle, assuming that the experimentally observed discrepancy is correct [9]. Many of these theories have been disproved, but there are two major surviving explanations which have not been conclusively refuted, but which also are not universally accepted.

The first explanation is that the presence of the muon so close to the proton deforms the proton, and that the radius we are measuring with muons is thus the radius of a deformed proton. This would be observed in an enhanced two-photon effect [18]. As the two-photon effect changes sign with lepton charge, observing the difference in cross section between opposite lepton charge states allows a direct observation of this effect.

The second explanation is that there are new electrophobic scalar bosons, a new force which interacts only with the muon and not the proton [19, 20,21]. This would be beyond-standard-model physics, breaking lepton universality. There are strong constraints placed on this proposal from other experiments, and thus the masses of the proposed particles are limited to a few- to tens-of$\mathrm{MeV}$. This would potentially also explain the muon g-2 anomaly. It could be seen in scattering data by observing modification in the muon-scattering cross section compared to the electron-scattering cross section around the invariant masses of these new particles.

\section{The MUon proton Scattering Experiment (MUSE)}

There have been spectroscopy measurements with electrons and muons, and scattering experiments with muons, but, to date, there has been no muon-proton elastic scattering data with sufficient precision to address the proton radius puzzle. MUSE, the MUon proton Scattering Experiment, was first proposed in 2012 to address this deficit. The MUSE detector setup can be seen in Figure 2. Full details of the MUSE experiment can be found in the MUSE TDR [7].

\subsection{The piM1 beam}

MUSE will run in the PiM1 beam line of the Paul Scherrer Institut in Villigen, Switzerland. PiM1 was constructed as a precision pion beam line, with the $590-\mathrm{MeV}$ proton beam of PSI impinging on a carbon target, and the resulting mixture of pions, electrons and muons being momentum-selected and transported to the experimental area by a precision magnet system. The magnet system polarity can be switched, giving MUSE the ability to measure in both charge states. Due to the mixed nature of the beam, MUSE has to identify the beam particles, particle-by-particle, using the relative timing between the accelerator RF and detectors in the experimental area. This does, however, mean that MUSE can simultaneously measure elastic scattering with both electrons and muons, reducing the systematic uncertainty in the comparison of both cross-sections. As the beam particles can subsequently decay in-flight, precise timing is needed at all stages of the experiment in order to remove decay backgrounds. In order to have a precisely defined scattering angle, using a secondary beam which has reasonably large dispersion and spot-size, MUSE has to track each individual beam particle on its way to the target. As the secondary beam has lower flux, it is necessary to use large-acceptance, non-magnetic spectrometers to detect scattered particles.

MUSE will run with both polarities at three different beam momenta of circa 115, 153 and $210 \mathrm{MeV} / \mathrm{c}$, giving six different data sets at overlapping $q^{2}$ (see Figure 1). We will use $3.3 \mathrm{MHz}$ of total beam flux, which, depending on momentum and polarity setting, will have a composition of $2-15 \% \mu, 10-98 \% e$, and $0-80 \% \pi$. In so doing, we will cover a $q^{2}$ range of $0.002-0.07 G e V^{2}$. 


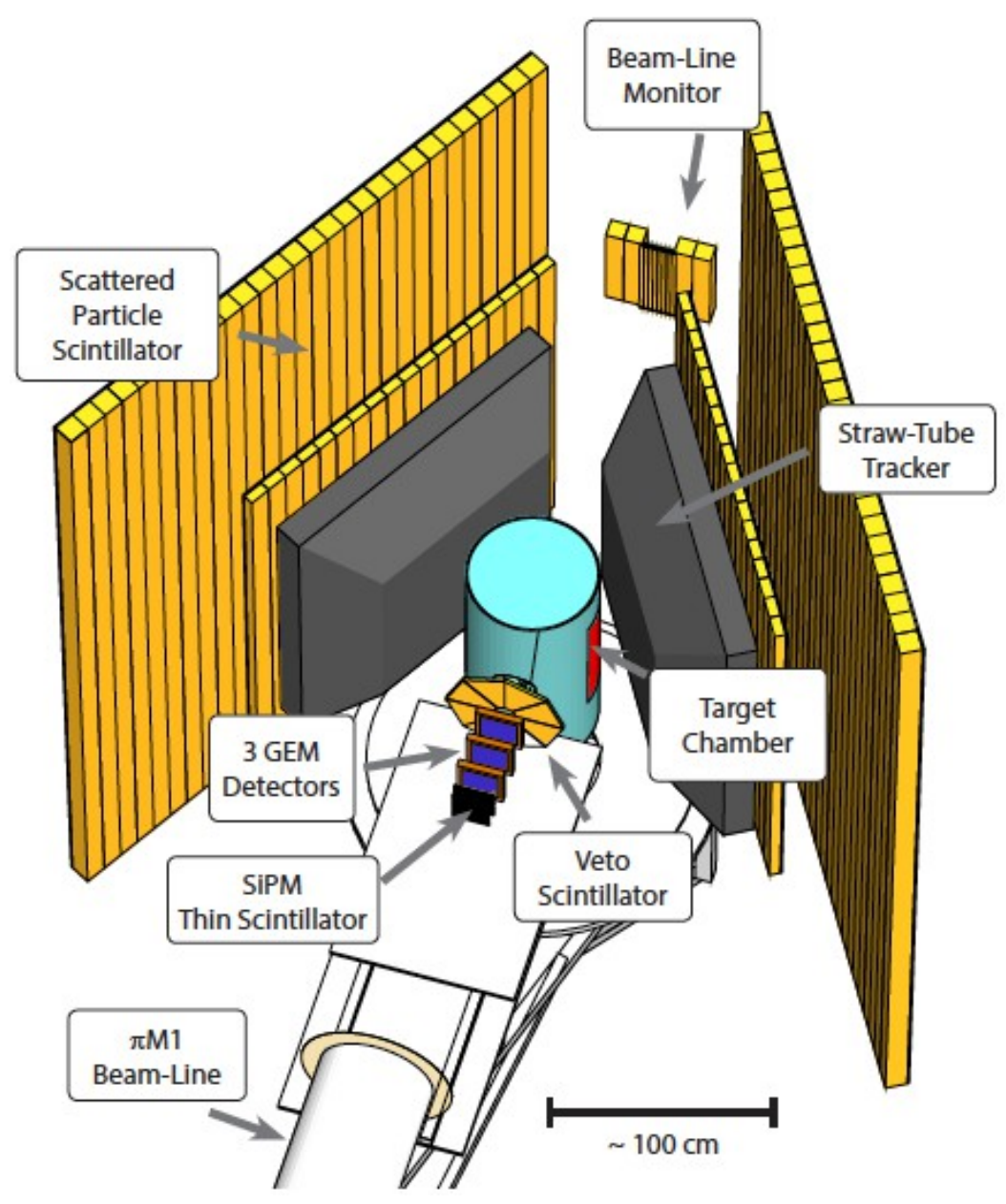

Figure 2: The MUSE detector system. The beam enters from the bottom left of the figure, and travels towards the upper right, exiting through the Beam-Line Monitor.

\subsection{Beam line detectors and target}

The first detector the beam encounters upon entering the PiM1 area is the Beam Hodoscope (BH): a series of thin, segmented 2mm-thick, BC-404 scintillators, read out at both ends by Hamamatsu S13360-3075PE silicon photo-multipliers. The planes each have six central bars which are $4 \mathrm{~mm}$ wide by $10 \mathrm{~cm}$ long. These are flanked on each side by five eight-mm-wide by $10-\mathrm{mm}$-tall bars, giving 16 bars per plane. The BH planes are alternated in $x$ - and $y$-orientations to give rough pixels. The number of $\mathrm{BH}$ planes employed depends on the beam momenta: at low momenta, less precise timing is needed and it is more important to minimize multiple scattering, so only two planes are used. At higher momenta, timing becomes more critical and multiple scattering less so, so four planes are used. The Beam Hodoscope is $99.8 \%$ efficient and has readout timing of better than 80 ps. The Beam Hodoscope was constructed by Tel Aviv University and Rutgers University.

Immediately after the $\mathrm{BH}$, there is a stack of three $10 \mathrm{~cm}$ by $10 \mathrm{~cm}$ Gas Electron Multiplier (GEM) detectors, which track the incoming beam with an efficiency of $98 \%$, and a position reso- 
lution of $70 \mu \mathrm{m}$. The GEMs were made for the OLYMPUS experiment [22], and are being reused in MUSE. They have an intrinsic angular resolution of $\sim 0.5 \mathrm{mr}$ and, when including the effects of multiple scattering, better than 5mr. Hampton University are responsible for the GEM detectors.

Following the GEMS, the beam passes into the liquid hydrogen target, designed by the University of Michigan group in collaboration with CREARE [23]. The target cell is a vertical Kapton cylinder, $6 \mathrm{~cm}$ in internal diameter, $11 \mathrm{~cm}$ high, filled with $280-\mathrm{ml}$ of liquid hydrogen. The vertically moving target ladder contains the filled cell; an identical, empty cell, for target cell background measurements; a thin carbon foil for tracking tests; a thin $\mathrm{CH} 2$ foil; and a blank position where no target cell is in the beam. The scattering chamber has two Kapton windows for beam entry and exit, and two large windows to allow scattered particles to reach the scattered particle detectors with minimum energy loss. These windows cover an azimuthal range of $20^{\circ}$ to $100^{\circ}$ on both sides of the beamline. They cover a polar angle of $-45^{\circ}$ to $+45^{\circ}$ at an azimuthal angle of $60^{\circ}$. In order to make strong but thin windows of this size ( $337 \mathrm{~mm}$ wide by $356 \mathrm{~mm}$ high) a sailcloth fabric which is made of Mylar ${ }^{\circledR}$ laminated on aramid fabric with an areal density of $368 \mathrm{~g} / \mathrm{m}^{2}$ was employed. The target is fully constructed and assembled at PSI, has passed all safety tests, and ran successfully with liquid hydrogen in late 2018 . It has a temperature stability at the 0.01-K level, suggesting a density stability at the $0.02 \%$ level.

Surrounding the entrance window to the target, is an annular ring of plastic scintillator, read out by SiPM detectors, which provides a VETO signal for the trigger. The VETO helps reduce the readout rate caused by beam particle decays or scattering from detectors upstream of the target.

Finally, all unscattered beam particles pass through the Beam Monitor (BM) detector, which is used to monitor beam position, flux and composition; and to perform separate time-of-fight measurements to verify the beam momentum. The beam monitor is formed of two planes of 16 thin scintillating elements read out by SiPMs, flanked on each side by two thick scintillator bars. The scintillator bars can be moved to the center of the detector for optimal timing resolution during the time-of-flight beam momentum measurements.

\subsection{Scattered particle detectors}

Scattered particles pass first through an array of two Straw Tube Tracking chambers (STTs) for tracking, then into a double wall of thicker scintillator bars used for timing and triggering of the scattered particles. The scattered particle detectors cover an azimuthal angle of $20^{\circ}$ to $100^{\circ}$ on both sides of the beamline. They cover a polar angle of $-45^{\circ}$ to $+45^{\circ}$ at an azimuthal angle of $60^{\circ}$.

The STTs follow the design made for the PANDA experiment [24]. They are made from thin-walled straws kept rigid by over-pressure, and are read out on a single end. The STTs are capable of resolving tracks with a resolution of $150 \mu \mathrm{m}$. The inner chambers are formed of five layers of 60 -cm-long straws orientated vertically, closest to the target, followed by five identical layers of straws orientated horizontally, moving radially outwards. The outer, 90-cm-long, straw chambers also have five horizontal, followed by five vertical, layers. The STTs were constructed by a collaboration between Hebrew University of Jerusalem, Israel, and Temple University.

Behind the STTs are two layers of Scattered Particle Scintillators (SPS). These are used to provide a fast scattered particle signal for triggering, and also to suppress backgrounds by providing fast timing. The bars, which are made from Eljen EJ-204 scintillator, are read out at both ends by Hamamatsu R13435 PMTs. This leads to an efficiency of 99\%, and a timing resolution of better 
than 55 ps. The design was based on the FToF12 for the CLAS12 JLab upgrade, and was developed and constructed by the University of South Carolina.

\subsection{Detector electronics and data acquisition system}

Timing of signals is the most crucial aspect of the MUSE readout, providing beam particle identification, and background suppression. To that end, we are reading out well over 3000 TDC channels, and 500 ADC channels, which are used to monitor gains and provide event-by-event walk corrections to improve timing in the SPS, BH and BM. All TDC signals and scalars are provided by TRB3 read-out boards [25], which are each equipped with 5 FPGAs, four peripheral FPGAs which read out individual channels, and one central FPGA which manages triggering, data-flow, and synchronization. Each TDC channel has an accompanying scalar, built into the TRB3 infrastructure. TRB3s are designed to provide a flexible, scalable, programmable readout system. Within MUSE, they provide timing and scalar information, and also programmed to provide an FPGA-based experiment trigger. The Data Acquisition System (DAQ) was constructed and programmed by George Washington University and the trigger by Rutgers University.

The input to the TRB3s comes from three different types of discriminator. The SPS is read out by PADIWA level discriminators, which are effective and inexpensive, designed by GSI to work with the TRB3 system [25]. The STTs faced large noise issues when they were first installed, and specially constructed PASTTREC cards were ordered, which have suppressed the noise issues, allowing the STTs to reach their designed resolution and efficiency [26]. The BH and BM face high rates and potential pile-up issues and, as such, are read out by MESYTEC MCFD-16s [27], which were specially adapted to output in LVDS format in order to better communicate with the TRB3s. To provide walk-corrections for the SPS, and to monitor performance of the BH and BM, Mesytec MQDC-32s [28] are employed to provide pulse-height information.

In order to trigger system readout, several TRB3s are programmed to provide an FPGA-based triggering system. The trigger requires that no pions, and at least one muon or electron was identified by timing in the Beam Hodoscope. The trigger further requires that the SPS identify a scattered particle which, based on a look-up table identifying acceptable combinations of front- and backSPS paddles, indicates a scattered particle originating in the hydrogen target region. A NOT of the veto, and a NOT of the full-system busy signal are also required for a trigger to be issued. The data are read out, and the overall DAQ system controlled by, MIDAS, a data-acquisition software authored by Stefan Ritt of PSI. Slow control and monitoring is performed in EPICS.

\subsection{MUSE status}

MUSE has full approval from the Paul Scherrer Institut BVR committee, which distributes beam time at the lab. The detector system is fully constructed and mounted on a movable, craneable platform. All detectors meet their design resolutions and efficiencies. Data taking begins in earnest with a summer 2019 beam time, during which MUSE will verify understanding of the beam: taking measurements to make systematic comparison between the data and simulations in both TURTLE and G4beamline; conclusively demonstrating that pion and muon momenta can be precisely determined in time-of-flight calibration measurements; and that MUSE can map out the relative momentum distributions of electron, muons, and pions in the beam. Once beam properties are fully verified, production data taking should begin in Fall 2019. 


\subsection{Anticipated precision of radius extraction}

The MUSE data analysis will be able to directly compare muon and electron cross sections, form factors, and radii. The first step will be to compare charge states: $\mu^{+} / \mu^{-}$and $e^{+} / e^{-}$to directly extract two-photon effects. Once these effects are fully determined, we will average over both charge states to eliminate two photon corrections and directly compare muons and electrons measured within the same experiment.

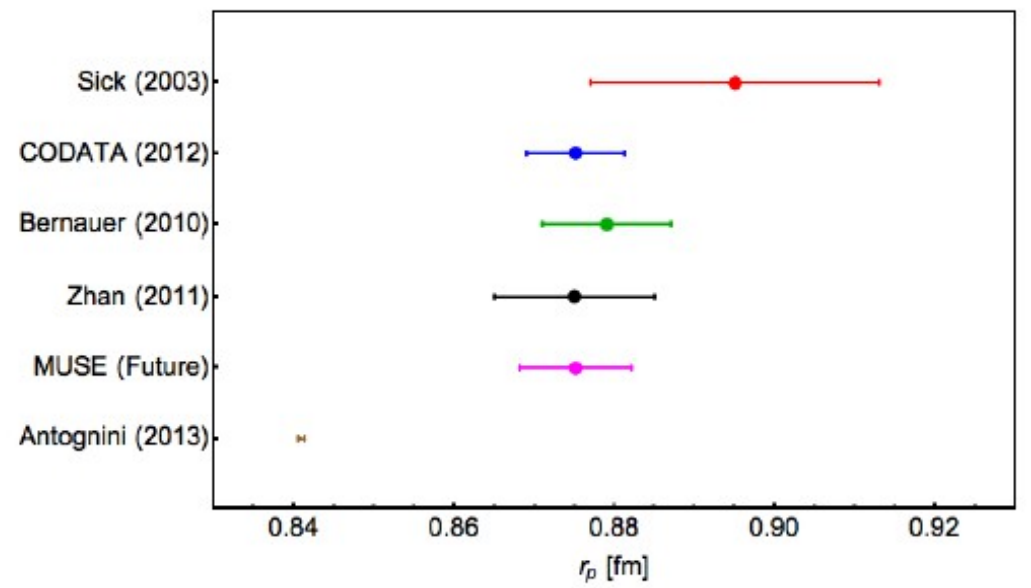

Figure 3: The predicted uncertainty in the absolute proton charge radius extraction from MUSE. The point is arbitrarily placed at $0.875 \mathrm{fm}$.

The statistical errors in the cross sections are planned to be better than $1 \%$ for backwards muons and much better for forward muons and electrons. We will be able to keep point-to-point relative uncertainties to a few tenths of a percent, allowing individual radius extractions from electrons and muons to $0.01 \mathrm{fm}$ (see Figure 3). By examining the ratio of electron and muon cross section data, we will be able to extract the radius difference to $0.005 \mathrm{fm}$ (Figure 4). If there is no difference, electron and muon data can be combined to extract the absolute radius to $0.007 \mathrm{fm}$.

The charge radius uncertainty is limited by systematics and fit uncertainties. MUSE detectors are complete and functioning at the required level to keep these effects under control, and many of the systematics, such as the target density, are vastly reduced, or completely eliminated by the simultaneous nature of the MUSE electron and muon measurements. Thus MUSE is suited to verify the $5.6 \sigma$ effect with even higher significance.

\section{Conclusion}

Almost a decade after the inception of the Proton Radius Puzzle, the puzzle endures. Muonic hydrogen, deuterium, and helium spectroscopy have further revealed this to be a $Z=1$ problem, rather than simply a proton radius problem. Recent high-precision hydrogen spectroscopy measurements have given conflicting radius values. Different fits to the same electron-scattering data sets give different radius values. In the midst of all of this, MUSE presents a unique opportunity to make the first measurement of elastic muon scattering on the proton with sufficient precision to address the proton radius. In addition to the precise muon scattering measurement, MUSE can make 


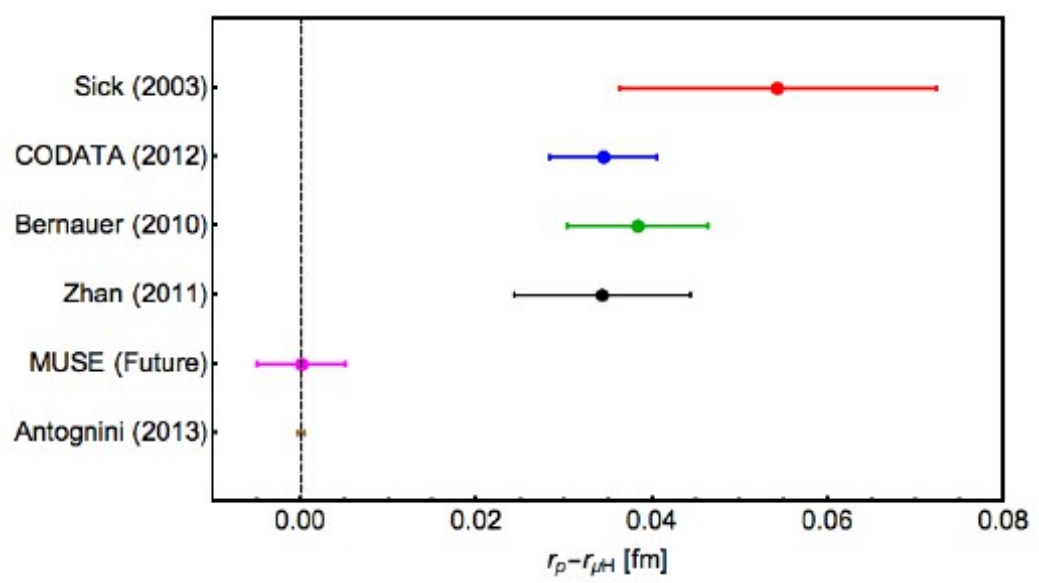

Figure 4: The predicted uncertainty in the proton charge radius difference from MUSE. The point is arbitrarily placed at $0.0 \mathrm{fm}$. Please note that all points other than MUSE show the difference between the published result and that of Antognini. MUSE shows the difference between MUSE proton and muon measurements.

a simultaneous electron measurement and subsequently access both charge states. This makes MUSE uniquely situated to provide fresh data with potential to resolve the proton radius puzzle.

\section{Acknowledgments}

This material is based upon work supported by the National Science Foundation under Grant Numbers PHY-1614850 and PHY-1714833.

\section{References}

[1] R. Pohl, A. Antognini, F. Nez, F. D. Amaro, F. Biraben et al., The size of the proton, Nature 466 (2010) 213.

[2] P. J. Mohr, B. N. Taylor and D. B. Newell, CODATA recommended values of the fundamental physical constants: 2010, Rev. Mod. Phys. 84 (2012) 1527.

[3] E. E. Chambers and R. Hofstadter, Structure of the Proton, Phys. Rev. 103 (1956) 1454.

[4] A1 Collaboration collaboration, High-precision determination of the electric and magnetic form factors of the proton, Phys.Rev.Lett. 105 (2010) 242001 [1007.5076].

[5] X. Zhan, K. Allada, D. Armstrong, J. Arrington, W. Bertozzi et al., High Precision Measurement of the Proton Elastic Form Factor Ratio $\mu_{p} G_{E} / G_{M}$ at low $Q^{2}$, Phys.Lett. B705 (2011) 59 [1102.0318].

[6] E. Kraus, K. E. Mesick, A. White, R. Gilman and S. Strauch, Polynomial fits and the proton radius puzzle, Phys. Rev. C 90 (2014) 045206.

[7] MUSE collaboration, Technical Design Report for the Paul Scherrer Institute Experiment R-12-01.1: Studying the Proton "Radius" Puzzle with $\mu$ p Elastic Scattering, https://arxiv.org/abs/1709.09753, 2017., . 
[8] V. Hill, G. Paz and R. Pohl, Workshop on the Precision Measurements and Fundamental Physics: The Proton Radius Puzzle and Beyond at the Mainz Institute for Theoretical Physics, https://indico.mitp.uni-mainz.de/event/132/, 2018.

[9] R. Pohl, R. Gilman, G. A. Miller and K. Pachucki, Muonic hydrogen and the proton radius puzzle, Annual Review of Nuclear and Particle Science 63 (2013) 175 [https://doi.org/10.1146/annurev-nucl-102212-170627].

[10] C. E. Carlson, The Proton Radius Puzzle, Prog. Part. Nucl. Phys. 82 (2015) 59 [1502. 05314$].$

[11] A. Antognini et al., Proton structure from the measurement of $2 s-2 p$ transition frequencies of muonic hydrogen, Science 339 (2013) 417.

[12] CREMA collaboration, Laser spectroscopy of muonic deuterium, Science 353 (2016) 669.

[13] A. Beyer, L. Maisenbacher, A. Matveev, R. Pohl, K. Khabarova, A. Grinin et al., The rydberg constant and proton size from atomic hydrogen, Science 358 (2017) 79 [http://science.sciencemag.org/content/358/6359/79.full.pdf].

[14] H. Fleurbaey, S. Galtier, S. Thomas, M. Bonnaud, L. Julien, F. Biraben et al., New Measurement of the $1 S-3 S$ Transition Frequency of Hydrogen: Contribution to the Proton Charge Radius Puzzle, Phys. Rev. Lett. 120 (2018) 183001 [1801.08816].

[15] Gasparian, Ashot, The prad experiment and the proton radius puzzle, EPJ Web of Conferences 73 (2014) 07006.

[16] D. Marchand, A new platform for research and applications with electrons: the PRAE project, EPJ Web Conf. 138 (2017) 01012.

[17] M. Mihovilovič et al., First measurement of proton's charge form factor at very low $Q^{2}$ with initial state radiation, Phys. Lett. B771 (2017) 194 [1612.06707].

[18] G. A. Miller, Proton Polarizability Contribution: Muonic Hydrogen Lamb Shift and Elastic Scattering, Phys. Lett. B718 (2013) 1078 [1209.4667].

[19] Y.-S. Liu, D. McKeen and G. A. Miller, Electrophobic scalar boson and muonic puzzles, Phys. Rev. Lett. 117 (2016) 101801.

[20] D. Tucker-Smith and I. Yavin, Muonic hydrogen and MeV forces, Phys. Rev. D 83 (2011) 101702.

[21] E. Izaguirre, G. Krnjaic and M. Pospelov, Probing New Physics with Underground Accelerators and Radioactive Sources, Phys. Lett. B740 (2015) 61 [1405. 4864 ].

[22] OLYMPUS collaboration, The OLYMPUS Experiment, Nucl. Instrum. Meth. A741 (2014) 1 [1312.1730].

[23] CREARE, 16 Great Hollow Rd, Hanover, NH 03755, USA., https://www.creare.com/, 2019.

[24] W. Erni et al., Technical design report for the PANDA (antiproton annihilations at darmstadt) straw tube tracker, The European Physical Journal A 49 (2013) 1.

[25] A. Neiser, J. Adamczewski-Musch, M. Hoek, W. Koenig, G. Korcyl, S. Linev et al., TRB3: a 264 channel high precision TDC platform and its applications, Journal of Instrumentation 8 (2013) C12043.

[26] M. Idzik, G. Korcyl, D. Przyborowski, P. Salabura, J. Smyrski and P. Strzempek, Readout electronics and data acquisition for gaseous tracking detectors, in 2016 IEEE-NPSS Real Time Conference (RT), pp. 1-3, June, 2016, DOI.

[27] Mesytec, MCFD-16, https://www.mesytec.com/products/datasheets/MCFD-16.pdf, 2019.

[28] Mesytec, MQDC-32, https://www.mesytec.com/products/datasheets/MQDC-32.pdf, 2019. 